\title{
Aesthetic Earlobe Reduction: A Practical Geometric Modification with Natural Contour Preservation
}

\author{
Sedat Tatar $^{1}$ Billur Sezgin ${ }^{1}$ \\ ${ }^{1}$ Department of Plastic, Reconstructive and Aesthetic Surgery, \\ Koc Universitesi, Istanbul, Turkey \\ Facial Plast Surg 2019;35:294-298.
}

\begin{abstract}
Address for correspondence Sedat Tatar, Department of Plastic Reconstructive and Aesthetic Surgery, Koc University Hospital, Davutpasa cad. No 4, Topkapi, 34010 Zeytinburnu, Istanbul 34450, Turkey (e-mail: sedattatarr@gmail.com).
\end{abstract}

\begin{abstract}
Keywords

- earlobe

- earlobe reduction

- earlobe ptosis

- earlobe reshaping

- earlobe surgery

Earlobe elongation is one of the signs of aging process and surgical correction is warranted for earlobe ptosis. Most of the earlobe reduction techniques result with scars on the anterior or inferior aspect of the earlobe or unnatural appearance. The authors present a modified technique for earlobe reduction and reshaping that results with an acceptable scar set on the natural groove of the anterior surface of the earlobe. Nine healthy female patients were operated for earlobe reduction. The postoperative and preoperative otobasion inferius (O)subaurale (S) distances of these patients were evaluated. According to the measurements, the preoperative mean $\mathrm{O}-\mathrm{S}$ distance of the right lobule was $13.1 \pm 1.8 \mathrm{~mm}$ and the left lobule was $12.8 \pm 1.9 \mathrm{~mm}$, while the postoperative $\mathrm{O}-\mathrm{S}$ distance of the right side was $3.4 \pm 1 \mathrm{~mm}$ and the left side was $3.4 \pm 0.7 \mathrm{~mm}$ (-Table 2). This translated to an approximate pre- and postoperative $\mathrm{O}-\mathrm{S}$ distance difference of $9 \mathrm{~mm}$, a significant reduction that resulted with ideal $\mathrm{O}-\mathrm{S}$ values. The presented approach has numerous advantages including inconspicuous scarring, smooth lateral earlobe contours, and undisturbed free borders. This was a Level IV therapeutic study.
\end{abstract}

Although the earlobe constitutes a small visible portion of the face during social interaction, deformities of the earlobe are often associated with unattractiveness. Congenital earlobe deformities are quite rare and are seen in 1:1500 live births with cleft earlobe being the most frequent presentation. Acquired earlobe deformities are much more frequently encountered and can be caused by trauma, surgery, utilization of gauging earrings, and aging. ${ }^{1}$

As with all tissues that undergo the aging process, the aging earlobe also presents itself with loss of elasticity, ptosis, wrinkling, and volume loss. The earlobe elongates $\sim 30$ to $35 \%$ with age and also presents with increased creasing. ${ }^{2}$ Nonsurgical interventions such as mesotherapy and the usage of dermal fillers are of benefit in the case of wrinkles and mild loss of elasticity associated with volume loss.

In the case of ptosis, however, surgical correction is warranted. A range of surgical techniques has been proposed to reshape and reduce the earlobe. However, most of these techniques result with scars on the anterior or inferior aspect of the ear lobule. Other postoperative distortions include dog-ear deformities, distortions on the subantitragal groove, or posterior notching which reduce the aesthetics of the ear lobule and ultimately result in decreased overall patient satisfaction. $^{3-6}$

Herein, we present a modified technique for earlobe reduction and reshaping that results with an acceptable scar set on the natural groove of the anterior surface of the earlobe.

\section{Material and Method}

Fifteen healthy female patients that presented consecutively to the plastic surgery outpatient clinic between March 2017 and March 2018 requesting the reduction and reshaping of their earlobes were recruited for this retrospective study. Written informed consent was obtained from all patients in the study. This study was designed in accordance with the national law and the World Medical Association Declaration of Helsinki (1964) with its ethical principles for medical research involving human subjects and subsequent amendments.

A detailed medical history was obtained from all patients prior to the procedure. Patients under the age of 18 years and published online May 17, 2019
Issue Theme Anything But the Knife: Nonsurgical Facial Plastic Surgery; Guest Editors: Deborah Watson, MD, FACS, and David B. Hom, MD, FACS
Copyright $\odot 2019$ by Thieme Medical Publishers, Inc., 333 Seventh Avenue, New York, NY 10001, USA. Tel: +1(212) 584-4662.
DOI https://doi.org/ 10.1055/s-0039-1688704. ISSN 0736-6825. 
those who were breastfeeding or pregnant were considered ineligible, while patients with chronic illnesses and those under treatment with medications, especially anticoagulants and corticosteroids were excluded from the study. The most important initial evaluation consisted of determining whether or not a patient was a good candidate for earlobe reduction. Patients that presented with isolated tissue atrophy or wrinkling without earlobe ptosis were referred for either hyaluronic acid-based tissue filler applications or fat injections. If earlobe ptosis was clinically determined through measurements, these patients were evaluated as good candidates for earlobe reduction surgery. Lateral earlobe piercings were approached with caution, and patients with gaping piercings were not operated due to the risk of circulatory compromise, while those with very small piercings were operated.

The classification system ${ }^{7}$ for earlobe ptosis previously described by Mowlavi et al was utilized in our study. The height of the earlobe was determined on the basis of anatomic landmarks, including the intertragal notch (I), the otobasion inferius $(\mathrm{O})$ (the caudal-most anterior attachment of the earlobe with the cheek skin), and the subaurale (S) (the caudal-most extension of the earlobe free margin) (-Fig. 1). The acceptable distance of the free caudal segment of the earlobe ( $\mathrm{O}-\mathrm{S}$ distance) was determined to be between 1 and $5 \mathrm{~mm}$, and any distance above $5 \mathrm{~mm}$ was considered to be unattractive. Therefore, patients with free caudal segments over $5 \mathrm{~mm}$ which translate to Grade II ptosis or higher were operated surgically for earlobe reduction.

\section{Surgical Technique}

Surgeries were done under local anesthesia or general anesthesia if additional procedures were performed. Regional infiltration of local anesthetics was limited to the base of the earlobe, to avoid distortion of the area to be incised.

The preoperative markings started $\sim 1$ to $2 \mathrm{~mm}$ below the $\mathrm{O}$ point depending on the patients' earlobe anatomy and

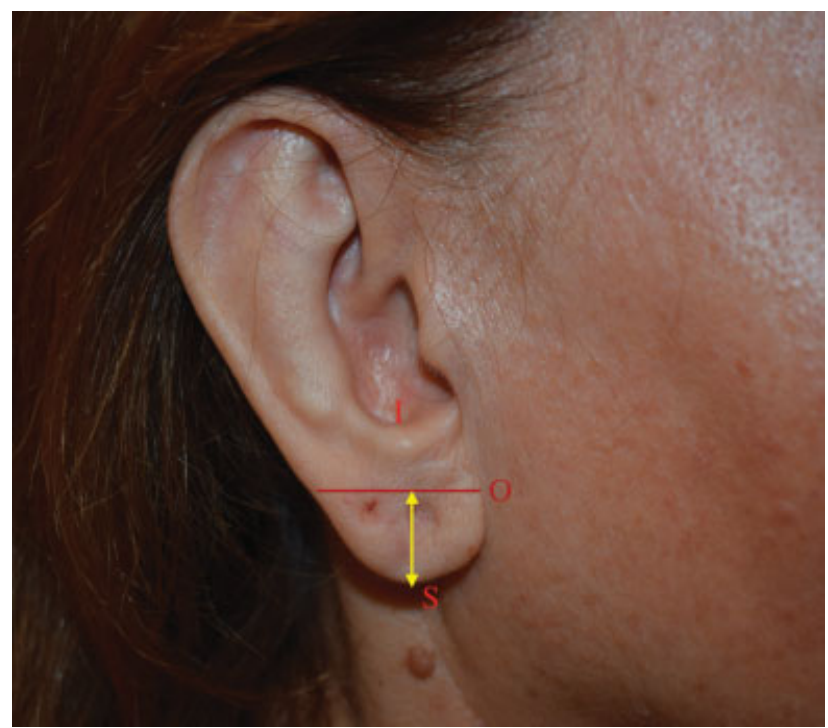

Fig. 1 Earlobe segments. Yellow line indicates O-S distance (I: intertragal notch, O: the otobasion inferius, S: subaurale). continued upward toward the intertragal notch until the level of the $O$ point was passed by 1 to $2 \mathrm{~mm}$ to point $A$. This maneuver allowed the earlobe to have a natural swoop at the cheek-earlobe interface. From this point A, the markings were continued in a slight curved line by following the normal groove of the anterior topography of the earlobe to point $B$, the pivot point. Care was given to preserve a width of at least 6 to $8 \mathrm{~mm}$ of tissue at the lateral border of the earlobe to avoid vascularization-related flap problems. Then a curved line with the same length was drawn at the medial margin of the earlobe flap that ended at point C. Finally, a final slightly curved line was drawn toward the caudal edge of the earlobe, depending on the amount of excision that was planned that ended at point $\mathrm{D}$. This line was drawn in similar length to the first line (-Fig. 2 ).

The incisions were made with a number 11 blade, taking care to cut through both the anterior and posterior surface of the earlobe simultaneously. The excision resulted with a double-crescentic defect in which points $A$ and $C$ coincided and the lateral earlobe flap fit in without any necessary modifications (-Fig. 3). Absorbable 5/0 polyglactin sutures were used for subcutaneous sutures, while skin closure was obtained with a few $6 / 0$ polypropylene sutures. Patients were instructed to use mupirocin ointment twice daily for 10 days, and suture removal was done on day 7 (-Fig. 4). The healing period was uneventful and no complications were encountered during follow-ups in any of the patients (-Figs. 5, 6, 7). Piercing holes were generally excised during surgery and patients were informed that they would require repiercing once healing was completed after 3 months. Patients were followed up 1 week and 3 months after surgery and the postoperative $\mathrm{O}-\mathrm{S}$ distance was measured at these time frames. Patients were also asked to rate their satisfaction regarding the appearance of their earlobe at the postoperative third month from a scale of 0 to 3 ( 0 being poor, 1 being no difference, 2 being better and 3 being much better).

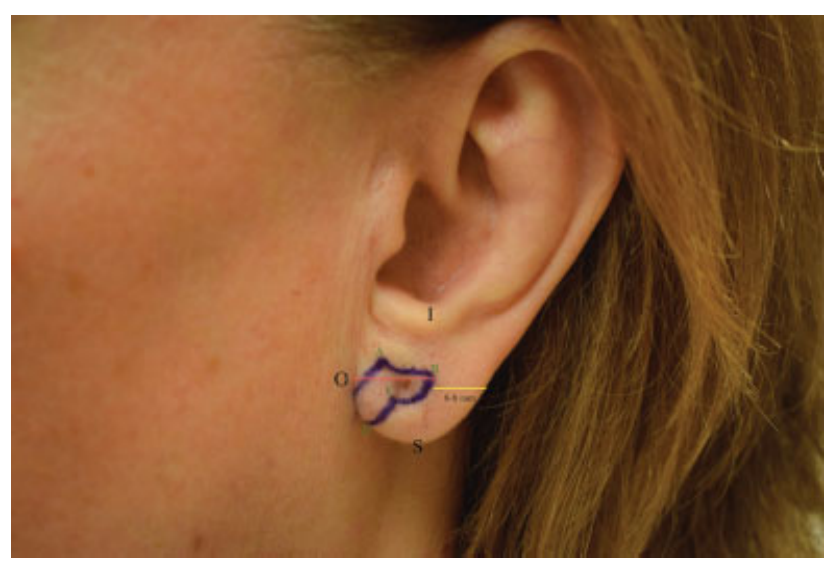

Fig. 2 A 62-year-old female patient with preoperative markings. Note that markings start 1 to $2 \mathrm{~mm}$ below the $O$ point and continue to the point $\mathrm{A}$, continues in a slight curved line by following the normal groove of the anterior topography of the earlobe to point $B$, the pivot point. Yellow line indicates preserved 6 to $8 \mathrm{~mm}$ of tissue at the lateral border of the earlobe. Curved line with the same length was drawn at the medial margin of the earlobe flap that ended at point $C$, a final slightly curved line ended at point $D$. 


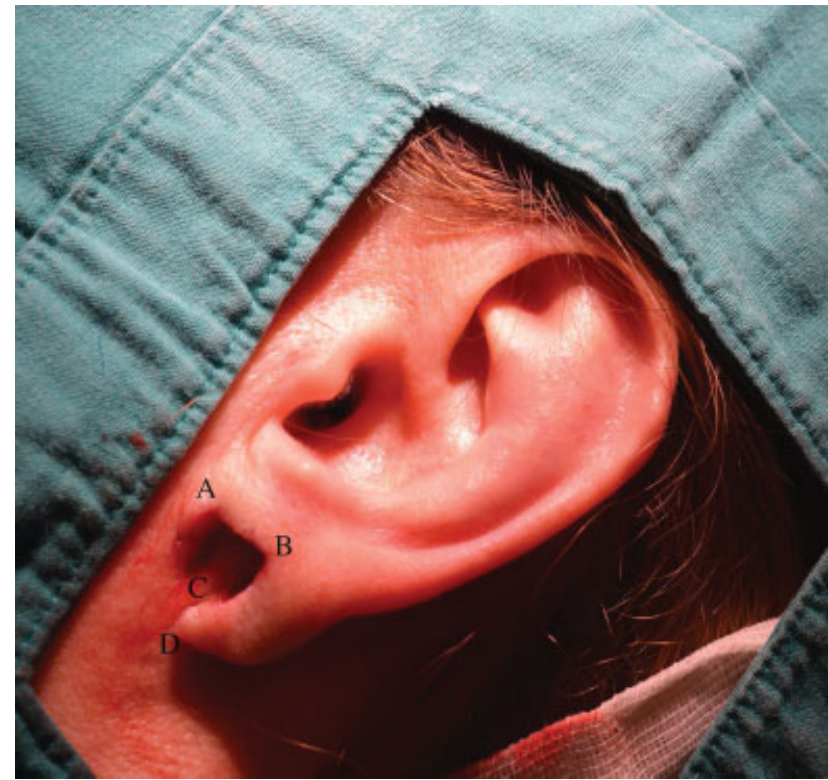

Fig. 3 Intraoperative view: the excision resulted with a double-crescentic defect in which points $A$ and $C$ coincided and the lateral earlobe flap fit in.

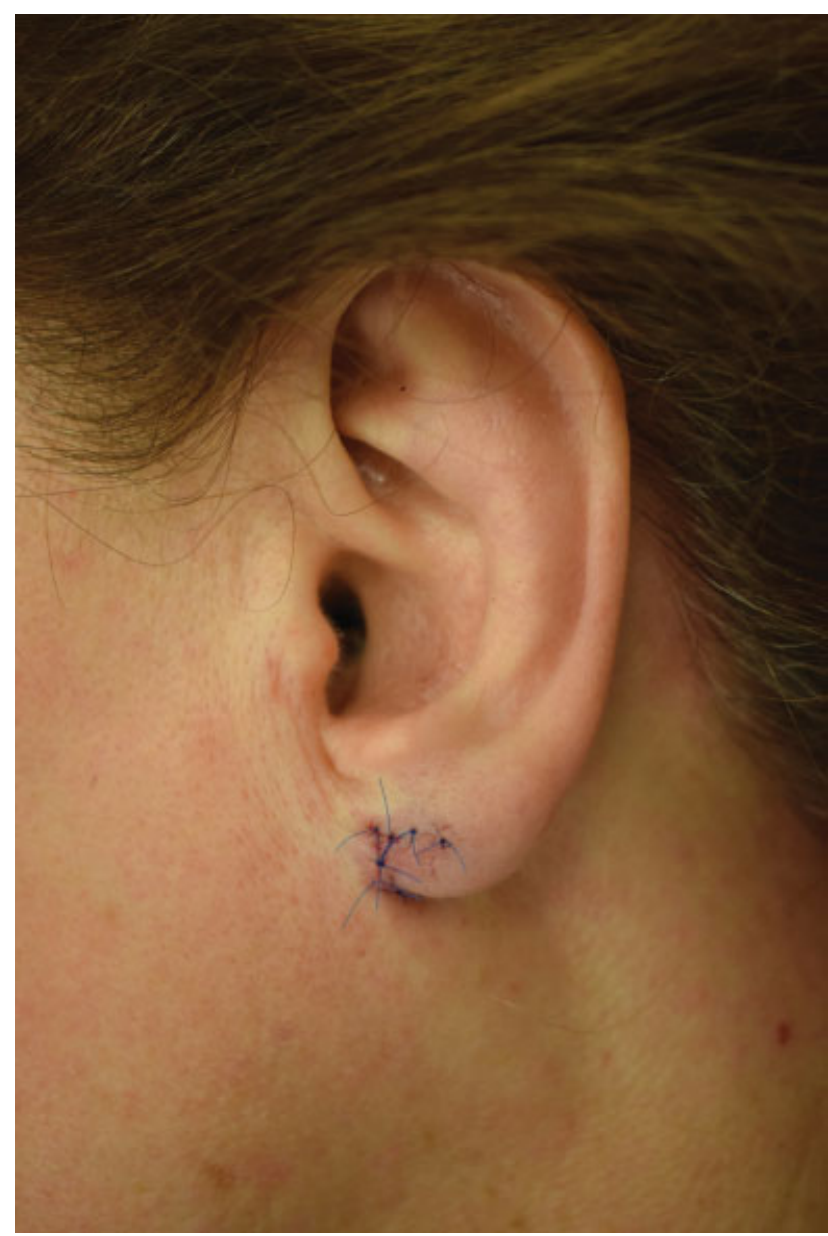

Fig. 4 Postoperative 1 week of the patient.

\section{Results}

Of the 15 patients seeking earlobe reshaping, 6 had isolated earlobe atrophy and wrinkling. These patients were deter-

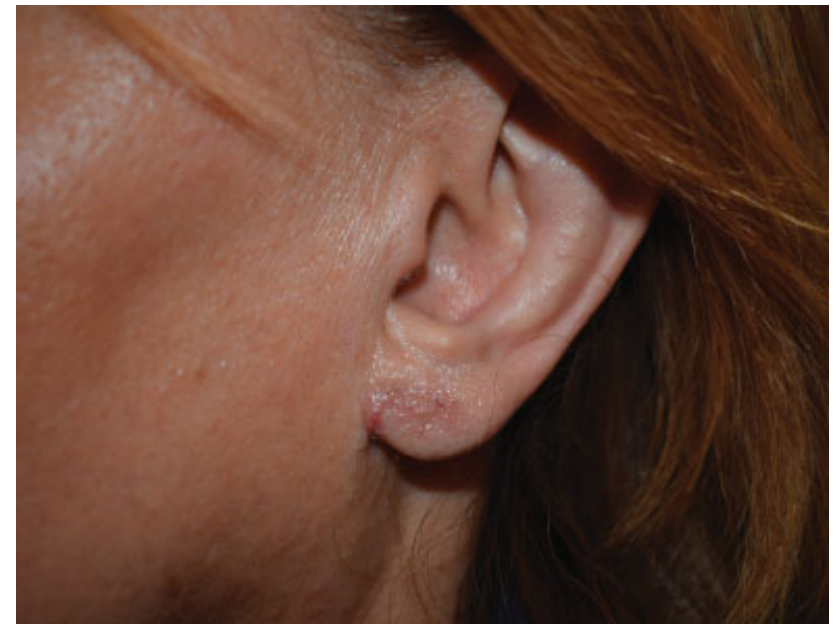

Fig. 5 Postoperative 30th day of the patients' earlobe.

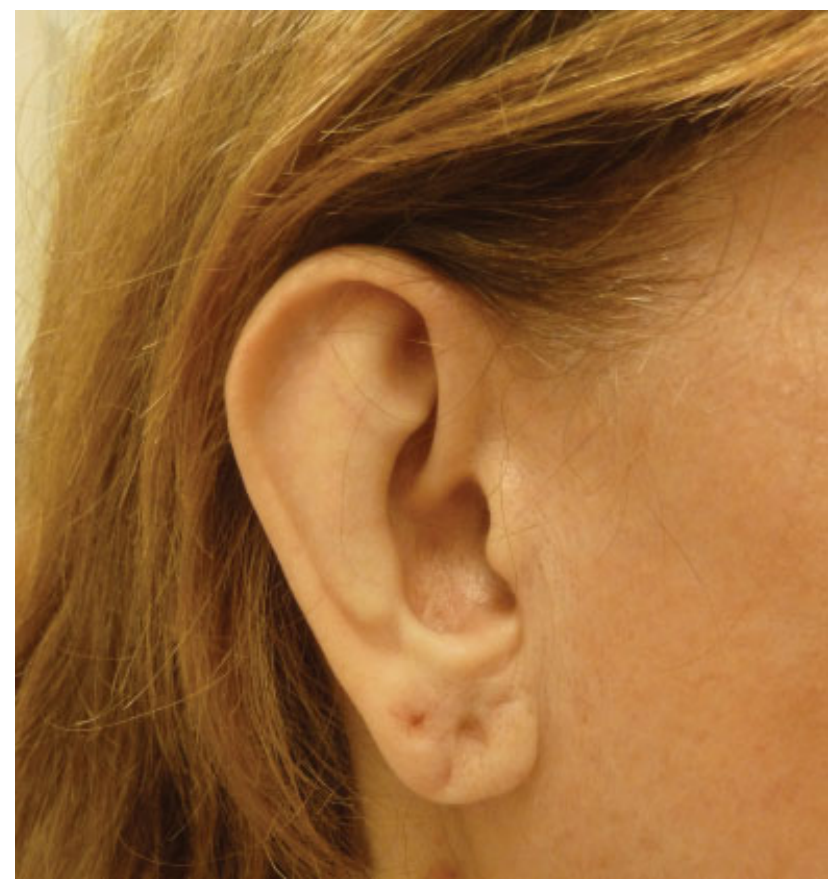

Fig. 6 Preoperative view of the right earlobe.

mined to be unsuitable for earlobe reduction surgery and following clinical evaluation, were treated with either tissue fillers or fat injection for voluminization.

A total of 18 earlobes were evaluated on 9 female patients who were operated on for earlobe ptosis. The mean age of the patients was 60.11 years (range; 52-67 years), while the mean follow-up period was 9.9 months (range; 6-14 months). Six patients only had earlobe reduction, while three patients had other procedures in combination with earlobe correction. These procedures were blepharoplasty, abdominoplasty, and breast lift.

The postoperative and preoperative $\mathrm{O}-\mathrm{S}$ distances of nine patients who underwent earlobe surgery were evaluated (-Table 1). According to the measurements, the preoperative mean $\mathrm{O}-\mathrm{S}$ distance of the right lobule was $13.1 \pm 1.8 \mathrm{~mm}$ 




Fig. 7 Postoperative 9th month of the patient's earlobe.

and the left lobule was $12.8 \pm 1.9 \mathrm{~mm}$, while the postoperative $0-S$ distance of the right side was $3.4 \pm 1 \mathrm{~mm}$ and the left side was $3.4 \pm 0.7 \mathrm{~mm}$ (-Table 2). This translated to an approximate pre- and postoperative $\mathrm{O}-\mathrm{S}$ distance difference of $9 \mathrm{~mm}$, a significant reduction that resulted with ideal $\mathrm{O}-\mathrm{S}$ values.

Patients were asked to rate their satisfaction in terms of the shape, suppleness, and overall appearance of their earlobes at the postoperative third month from a scale of 0 to 3 . According to this, seven patients $(77.7 \%)$ stated that their earlobes were much better, while two patients $(22.2 \%)$ stated that the appearance of their earlobes following reduction was better.
Table 2 Preoperative and postoperative mean O-S distances

\begin{tabular}{|l|l|l|}
\hline Mean O-S distance (mm) & Preoperative & Postoperative \\
\hline Right & $13.1 \pm 1.8$ & $3.4 \pm 1$ \\
\hline Left & $12.8 \pm 1.9$ & $3.4 \pm 0.7$ \\
\hline
\end{tabular}

Abbreviation: OS, otobasion inferius (O)-subaurale (S) distances.

\section{Discussion}

The size and orientation of the ear along with congenital and acquired ear deformities have been thoroughly studied in literature, but minimal attention has been directed to the shape and size of the ear lobule., ${ }^{1,2}$ Besides trauma and iatrogenic or congenital deformities, aging is an important factor that causes earlobe deformity. The aging earlobe frequently presents with elongation or ptosis, along with volume loss and excessive creasing. ${ }^{8}$

Several surgical techniques have been described to address the changes in the aging earlobe. These techniques range from simple wedge excision to complex geometrical designs and each technique comes with advantages and limitations. ${ }^{4-6}$ Simple wedge excision for the reduction in the earlobe was first introduced by Miller in 1925 followed by several other geometric excisional techniques for earlobe correction..$^{9-11}$ Although these techniques succeeded in decreasing the length and width of the earlobe, the main disadvantages were prominent anterior scarring and unnatural creasing. To avoid prominent anterior scars, elliptical skin excision from the free inferior edge of the earlobe was introduced by Stark and Mccoy, ${ }^{12,13}$ a technique that is still widely practiced by plastic surgeons. Although the scar is hidden on the inferior and posterior aspect of the lobule, there is a high probability of distortion at the free lateral edge with this method.

Recently, a technique by Van Putte and Colpaert has been described for earlobe reduction which follows general geometric principles and results with undisturbed free borders and ideal anterior scar locations. ${ }^{14}$ Our technique shares the

Table 1 Preoperative and postoperative measurements of O-S distance

\begin{tabular}{|l|l|l|l|l|}
\hline & O-S distance right ear $(\mathrm{mm})$ & O-S distance left ear (mm) \\
\hline Patient no. & Preoperative & Postoperative & Preoperative & Postoperative \\
\hline 1 & 16 & 3 & 15 & 3 \\
\hline 2 & 13 & 2 & 13 & 2 \\
\hline 3 & 11 & 4 & 10 & 4 \\
\hline 4 & 14 & 5 & 13 & 4 \\
\hline 5 & 12 & 4 & 12 & 4 \\
\hline 6 & 15 & 2 & 14 & 3 \\
\hline 7 & 14 & 4 & 15 & 4 \\
\hline 8 & 12 & 3 & 13 & 10 \\
\hline 9 & 11 & 4 & & 3 \\
\hline
\end{tabular}

Abbreviation: OS, otobasion inferius (O)-subaurale (S) distances. 
advantage of an untouched lateral edge with this technique. The maneuver of transposing a superior pedicled lateral earlobe flap prevents unsightly notching in this area. The main difference of our technique from the latter method is that our incision starts 1 to $2 \mathrm{~mm}$ below the 0 point and preserves the earlobe-cheek skin interface to provide a natural swoop in the medial portion of the earlobe while preventing rotation and traction following surgical correction. Another advantage of our technique is that the free edge of the lateral earlobe flap can be angled according to each patient's helical and conchal anatomy where preserving more tissue results with a wider earlobe and less distal tissue results with a less-wider earlobe. This allows the surgeon to reduce the earlobe in both the vertical (height) and horizontal (width) directions by adjusting the excision depending on which dimension is more problematic. As the antitragal groove is a slightly curved anatomical landmark, the resulting scar with this technique is also a slightly curved one located right under the natural groove, resulting with a less conspicuous scar. Although none of the procedures were done in combination with facelift surgery, preserving the small amount of tissue at the earlobe-cheek interface and thereby separating the incisions of rhytidectomy and earlobe reduction can be a very beneficial tool in preventing the frequent manifestation of pixie ear deformity caused by traction during the healing phase.

We approached lateral earlobe piercings with caution as compromise in the circulation of the lateral flap can be encountered in patients with widened holes at the lateral aspect. This is also a potential issue for patients that present with earlobe ptosis due to gauging piercings, in which another surgical technique should be opted for to minimize the risk of lateral flap necrosis. ${ }^{15}$

In conclusion, the presented approach has numerous advantages including inconspicuous scarring, smooth lateral earlobe contours, and undisturbed free borders.

Preservation of minimal tissue at the cheek-earlobe interface prevents traction and provides a natural swoop in the medial portion of the earlobe with optimal shape that yields high patient satisfaction.

\section{Conflicts of Interest}

The authors declare that they have no conflicts of interest to disclose.

\section{References}

1 Zhao H, Ma L, Qi X, et al. A morphometric study of the newborn ear and an analysis of factors related to congenital auricular deformities. Plast Reconstr Surg 2017;140(01):147-155

2 Azaria R, Adler N, Silfen R, Regev D, Hauben DJ. Morphometry of the adult human earlobe: a study of 547 subjects and clinical application. Plast Reconstr Surg 2003;111(07):2398-2402, discussion 2403-2404

3 Mowlavi A, Meldrum DG, Wilhelmi BJ, Zook EG. Effect of face lift on earlobe ptosis and pseudoptosis. Plast Reconstr Surg 2004;114 (04):988-991

4 Lassus $C$. Another technique for the reduction of the earlobe. Aesthetic Plast Surg 1982;6(01):43-45

5 Connell BF. Correcting deformities of the aged earlobe. Aesthet Surg J 2005;25(02):194-196

6 Tipton JB. A simple technique for reduction of the earlobe. Plast Reconstr Surg 1980;66(04):630-632

7 Mowlavi A, Meldrum DG, Wilhelmi BJ, Ghavami A, Zook EG. The aesthetic earlobe: classification of lobule ptosis on the basis of a survey of North American Caucasians. Plast Reconstr Surg 2003; 112(01):266-272, discussion 273-274

8 Mowlavi A, Meldrum DG, Kalkanis J, Wilhelmi BJ, Russell RC, Zook EG. Surgical design and algorithm for correction of earlobe ptosis and pseudoptosis deformity. Plast Reconstr Surg 2005;115(01):290-295

9 Tanzer RC. Congenital deformities of the auricle. In: Converse JM, ed. Reconstructive Plastic Surgery. 2nd edition. Philadelphia: Saunders; 1977:1719

10 Miller CC. Cosmetic Surgery: The Correction of Facial Features and Imperfections. 2nd edition. Philadelphia: FA Davis; 1925

11 Loeb R. Correcao da hypertrofia da lobulo auricular. Rev a. Lat Am Chir Plast 1965;9:186

12 Stark RB. Plastic Surgery. New York: Hoeber Medical Division, Harper and Row; 1962:256

13 McCoy FJ. Macrotia. In: Masters FW, Lewis JR, eds. Symposium on Aesthetic Surgery of the Nose, Ears and Chin. St. LouisC.V. Mosby 1973:162

14 Van Putte L, Colpaert SD. Earlobe reduction with minimally visible scars: the sub-antitragal groove technique. Aesthetic Plast Surg 2017;41(02):335-338

15 Brothers RJ, Jahnke MN, Daveluy S. Exaggerated earlobe ptosis due to habitual ear pulling. JAMA Dermatol 2014;150(06):673-674 